\title{
PHOTOMETRIC PERIODICITIES IN MAGELLANIC CLOUD BE/NEUTRON STAR BINARIES
}

\author{
P. C. Schmidtke, ${ }^{1}$ A. P. Cowley, ${ }^{1}$ Lance Levenson, ${ }^{1}$ and Katie Sweet ${ }^{1}$
}

\begin{abstract}
We are investigating the photometric properties of $\mathrm{Be} /$ neutron star systems in the Magellanic Clouds using $V, R$, and $I$ data from the MACHO and OGLE-II surveys. Here we give results for 6 systems, 4 of which are $\mathrm{X}$ ray pulsars.
\end{abstract}

The long-term light curves for each of these systems were searched for periodic variations. We first pre-whitened the data by fitting an appropriate polynomial to "flatten" lengthy portions of the light curves. We then used the method described by Horne \& Baliunas (1986) to find periodicities in the relative magnitudes. In each case we found significant power at what we interpret as the orbital period $\left(\mathrm{P}_{\text {orb }}\right)$. The folded light curves show small outbursts, usually with rapid brightening and more gradual decline. If the orbits are eccentric (as in the classical case of A0535-66, e.g. Charles et al. 1983), the periodic brightening occurs each time the neutron star approaches periastron and passes through the equatorial disk of the Be star.

We have also searched the data between orbital outbursts for other periodic variability $\left(\mathrm{P}_{2}\right)$. In 3 of the systems we find secondary variations that are quasi-periodic, where $\mathrm{P}_{2}$ varies by $\sim 10 \%$ between segments. We propose that these optical QPO-like variations (' $Q P V$ ') may be caused by a changing view of the brightened region in the disk due to its rotation around the Be star. If the disk size is not constant with time, then secular changes are expected in this rotation period.

For each system we list our photometric $\mathrm{P}_{\text {orb }}, \mathrm{P}_{2}$ (QPV found between orbital outbursts), and amplitudes. Spin periods $\left(\mathrm{P}_{\text {pulse }}\right)$ are also given for the $4 \mathrm{X}$-ray pulsars. These binaries fit the known relationship between $\mathrm{P}_{\text {pulse }}$ and $\mathrm{P}_{\text {orb }}$ on the "Corbet diagram" (Corbet 1984).

RX J0049.4-7323: This binary has X-ray pulses with $\mathrm{P}_{\text {pulse }}=755.5 \mathrm{~s}$ (Yokogawa et al. 2000). The long-term light curves show $\sim 0.3$ mag outbursts every 394 days (probably $\mathrm{P}_{\text {orb }}$ ). Analysis of the data between outbursts shows QPV with $\mathrm{P}_{2} \sim 10.9 \mathrm{~d}$ and
$\Delta R \sim 0.05$ (Cowley \& Schmidtke 2003).

AX J0051-722: X-ray data show $P_{\text {pulse }}=91$.1s (Corbet et al. 1998). Optically, only MACHO photometry is available. Period analysis yields $\mathrm{P}_{\text {orb }}=88.25 \mathrm{~d}$ with $\Delta R, V \sim 0.04$. This system also shows QPV at $\mathrm{P}_{2} \sim 6.2 \mathrm{~d}$ with $\Delta V \sim 0.04$, making the two periods difficult to separate.

CXOU J005736.2-721934: Chandra X-ray data show this source has $\mathrm{P}_{\text {pulse }}=564.8 \mathrm{~s}$ ( $\mathrm{Nacomb}$ et al. 2003). Optical photometry reveals $\mathrm{P}_{\text {orb }}=95.3 \mathrm{~d}$, with $\Delta R \sim 0.04$. $Q P V$ are present at $\mathrm{P}_{2} \sim 4.9 \mathrm{~d}$ and $\Delta R \sim 0.03$.

RX J0058.2--7231: The orbital period of $59.72 \mathrm{~d}$ is evident to the eye in the long-term light curve. It shows an amplitude of $\Delta I \sim 0.08$ and $\Delta R \sim 0.06$ (Schmidtke et al. 2003). However, a search for additional periods did not reveal any QPV. This system is not known to be an X-ray pulsar.

RX J0101.3-7211: XMM data show $\mathrm{P}_{\text {pulse }}$ $=455 \mathrm{~s}$ (Sasaki et al. 2001). OGLE/MACHO light curves reveal $\mathrm{P}_{\text {orb }}=74.7 \mathrm{~d}$ with $\triangle I \sim 0.1$ and $\triangle R, V \sim 0.04$. QPV were not found.

RX J0520.5-6932: Coe et al. (2001) found $\mathrm{P}_{\text {orb }}=24.44 \mathrm{~d}$ from OGLE data. Analyzing the larger MACHO data set, we find the same period in both $R$ and $V$ with amplitudes of $\sim 0.02-0.03$. QPV may be weakly present at $3.2 \mathrm{~d}$. This weak $\mathrm{X}$-ray source has no known X-ray pulses.

\section{REFERENCES}

Charles, P.A., et al. 1983, MNRAS, 202, 657

Coe, M.J., et al. 2001, MNRAS, 324, 623

Corbet, R.H.D. 1984, A\&A, 141, 91

Corbet, R., et al. 1998, IA IC C. No. 6803

Cowley, A.P., \& Schmidtke, P.C 2003, - 1J in press

Horne, J.H., \& Baliunas, S.L. 1986, ApJ, 302, 757

Macomb, D.J., et al. 2003, ApJ, 58.4. L79

Sasaki, M., et al. 2001, A\&A, 369, L32

Schmidtke, P.C., Cowley, A.P., \& Lerenson, L. 2003, A.J, 126,1017

Yokogawa, J., et al. 2000, PASJ, 52, L73

\footnotetext{
${ }^{1}$ Dept. Physics and Astronomy, Arizona State Univ., Tempe, AZ 85287-1504, USA.
} 